\title{
Spectrum to distance mapping via nonlinear Airy pulses
}

\author{
Yi Hu, ${ }^{1,2}$ Ming Li, ${ }^{1}$ Domenico Bongiovanni, ${ }^{1}$ Matteo Clerici,${ }^{1,3}$ Jianping Yao, ${ }^{5}$ Zhigang Chen,${ }^{2,4}$ \\ José Azaña, ${ }^{1}$ and Roberto Morandotti ${ }^{1, *}$ \\ ${ }^{1}$ Institute National de la Recherche Scientifique-EMT, 1650 Blvd. Lionel-Boulet, Varennes, Quebec J3X 1S2, Canada \\ ${ }^{2}$ The Key Laboratory of Weak-Light Nonlinear Photonics, Ministry of Education and TEDA Applied Physics School, \\ Nankai University, Tianjin 300457, China \\ ${ }^{3}$ School of Engineering and Physical Sciences, Heriot-Watt University, Edinburgh EH14 4AS, UK \\ ${ }^{4}$ Department of Physics \& Astronomy, San Francisco State University, San Francisco, California 94132, USA \\ ${ }^{5}$ School of Information Technology and Engineering, University of Ottawa, Ottawa, Ontario K1N6N5, Canada \\ *Corresponding author: morandot@emt.inrs.ca
}

Received November 20, 2012; revised December 18, 2012; accepted January 2, 2013; posted January 3, 2013 (Doc. ID 180239); published February 1, 2013

\begin{abstract}
We theoretically and experimentally study the phenomena related to self-phase modulation of Airy pulses in fibers. During nonlinear evolution, most spectral components of the Airy pulses concentrate into one or two peaks for normal and anomalous dispersion, respectively. The resulting peaks self-shift along the propagation, effectively mapping the longitudinal coordinate into the frequency domain. The frequency shift can be precisely controlled by simply acting on the spectral cubic phase structure without the need to alter the fiber length.
\end{abstract}

Airy pulses are self-accelerating optical wave packets featured by quasi-nondispersive intense peaks [ [1-7]. In linear optics, they are the key ingredients for the realization of optical bullets [2,3], while in a nonlinear environment, they tend to shed off-shooting solitons under the effect of a Kerr nonlinearity [6]. They can also be exploited to control super-continuum generation in highly nonlinear fibers [7]. Such Airy pulses are analogous to spatial Airy beams $[1,8]$, which have been studied extensively due to their intriguing phenomena and potential applications [9]. One of the several properties of Airy beams is the appearance of a spectral defect in the spatial spectrum that shifts along the propagation in media under saturable nonlinearity [10]. We expect the temporal counterpart of this effect to be a spectral feature that varies along the propagation length.

In this Letter, we study the self-phase modulation (SPM) of Airy pulses under the effect of both anomalous and normal dispersion in fibers. These self-accelerating pulses are generated through the modulation of Gaussian pulses via the cubic spectral phase structures produced in a pulse shaper. We consider the nonlinear propagation of an optical pulse in a fiber for a simplified but realistic condition in which the Kerr effect is the only active nonlinearity while solely the second order dispersion term $\left(\beta_{2}\right)$ is assumed as relevant. Under these assumptions, the pulse evolution is described by a nonlinear Schrödinger equation that, in a normalized notation, reads as [11]:

$$
\partial \varphi / \partial \xi=-0.5 i \operatorname{sgn}\left(\beta_{2}\right) \partial^{2} \varphi / \partial T^{2}+i \eta|\varphi|^{2} \varphi
$$

where $\xi$ and $T$ are the normalized distance and relative delay (with respect to the group delay). In terms of physical distance $z$ and time $t$, one unit of $\xi$ and $T$ equals $z\left|\beta_{2}\right| / t_{0}^{2}$ and $\left(t-z / v_{g}\right) / t_{0}$, respectively, where $t_{0}$ is an arbitrary time scaling factor and $v_{g}$ is the pulse group velocity. In Eq. (1), $\operatorname{sgn}(\cdot)$ is the sign operator, $\varphi$ is the electrical field envelope, and $\eta=\gamma /\left(t_{0}^{2}\left|\beta_{2}\right|\right)$ corresponds to the nonlinear parameter $\gamma$ in fibers.
Before studying the nonlinear dynamics of Airy pulses using Eq. (1), let us briefly recall how SPM affects the propagation of a chirped Gaussian pulse given by $\exp \left[\left(-\alpha v^{2}+i \operatorname{sgn}\left(\beta_{2}\right) v^{2}\right]\right.$ ( $\alpha$ determines the bandwidth and $v$ is the normalized angular frequency). Due to the linear temporal focusing induced by the combination of chirp and dispersion, the maximum peak power (MPP) for these pulses appears inside the fibers. Figure 1 shows the nonlinear spectral reshaping of positively or negatively chirped Gaussian pulses under the effect of (a) an anomalous $\left(\beta_{2}<0\right)$ and (b) a normal $\left(\beta_{2}>0\right)$ dispersion, in the case of a mild nonlinearity. The spectra reshape dramatically near the MPP location and for $\beta_{2}<0$ and $\beta_{2}>0$, a hole and a peak appear in the pulse spectrum, respectively [Figs. 1(a) and 1(b)].

With this in mind, we now analyze the SPM of Airy pulses. Such pulses are associated with a spectral cubic phase $\exp \left[-i\left(\omega / \omega_{s}\right)^{3} / 3\right]$ ( $\omega$ is the angular frequency and $\omega_{s}$ is a scaling factor). By letting $t_{0}=1 / \omega_{s}$, this phase simply becomes $\exp \left(-i v^{3} / 3\right)$ in the normalized coordinates. First, we consider the linear dynamics of Airy pulses (i.e., $\eta=0)$. The spectral amplitude $\exp \left(-\alpha v^{2}\right)$ of the input Gaussian pulse is modulated by a cubic phase $\exp \left[-i\left(v-v_{m}\right)^{3} / 3\right]\left(v_{m}\right.$ denotes the offset). The linear propagation can then be described analytically by
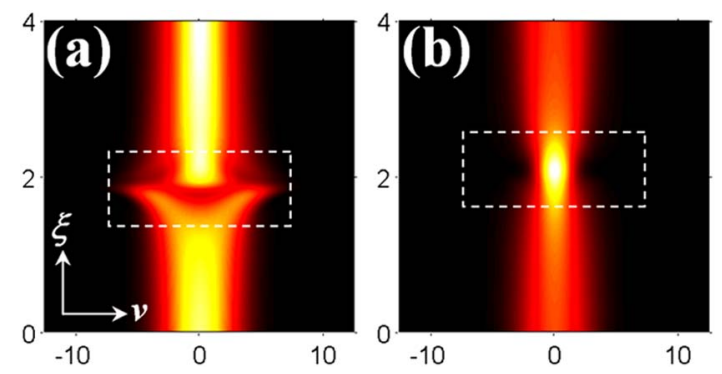

Fig. 1. (Color online) Typical spectral evolution of a Gaussian pulse propagating in an optical fiber in the presence of a mild nonlinearity for (a) anomalous and (b) normal dispersion. 


$$
\begin{aligned}
\varphi(T, \xi)= & A i\left[T-\varsigma^{2} / 4+\alpha^{2}+v_{m}^{2}-i \operatorname{sgn}\left(\beta_{2}\right) \alpha \varsigma\right] \\
& \times \sqrt{2 \pi} \exp \left(-i v_{m}^{3} / 3\right) \exp \left(\alpha T-i \operatorname{sgn}\left(\beta_{2}\right) \varsigma T / 2\right) \\
& \times \exp \left[i 2\left(i \alpha+\operatorname{sgn}\left(\beta_{2}\right) \varsigma / 2\right)^{3} / 3\right. \\
& \left.-i v_{m}^{2}\left(i \alpha+\operatorname{sgn}\left(\beta_{2}\right) \varsigma / 2\right)\right],
\end{aligned}
$$

where $\varsigma=\xi+2 v_{m} / \operatorname{sgn}\left(\beta_{2}\right)$. From Eq. (2), we readily obtain that the Airy pulse follows a parabolic time trajectory:

$$
T_{p}=\varsigma^{2} / 4-v_{m}^{2}-\alpha^{2} .
$$

When $v_{m}=0$, the linear dynamics of the Airy pulse can be analogous to that in the spatial domain [1] . In this case, the MPP appears at the input. From Eq. (2), one can notice that by adding a frequency offset $v_{m}$ into the cubic phase term (basically introducing a quadratic phase), the whole linear dynamics of the Airy pulse can be delayed or advanced, and accordingly the MPP is located at $\xi=-2 v_{m} / \operatorname{sgn}\left(\beta_{2}\right)$.

To avoid the breakdown of accelerating Airy wavepackets typically induced by a nonlinearity, the MPP is set inside a fiber $(\xi=8)$ by choosing $v_{m}=-4 \operatorname{sgn}\left(\beta_{2}\right)$ $[\underline{6}, \underline{10}, \underline{12}]$. As we know, the Kerr effect becomes significant for high enough peak powers. For Airy pulses, the nonlinearity is hence mainly induced by the first intense hump propagating along the time trajectory described by Eq. (3). Figure 2(a) shows a section of the main hump with an instantaneous power larger than half of the MPP. In order to understand the nonlinear dynamics, we model the propagation of the intense lobe as a Gaussian pulse featured by a linear phase term varying along propagation: $\exp \left[-i \operatorname{sgn}\left(\beta_{2}\right) \mu T\right]$, where $\mu=$ $d T_{p} / d \xi=\xi / 2+v_{m} / \operatorname{sgn}\left(\beta_{2}\right)$. This linear phase term in the time domain corresponds to a carrier frequency shift, i.e., the instantaneous frequency of the intense lobe varies linearly along the pulse propagation:

$$
v_{d}=\operatorname{sgn}\left(\beta_{2}\right) \mu=\operatorname{sgn}\left(\beta_{2}\right) \xi / 2+v_{m} .
$$
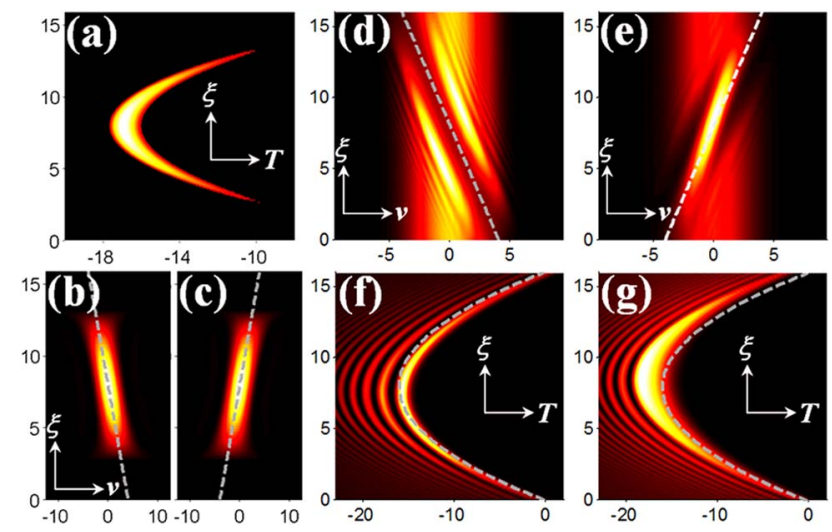

Fig. 2. (Color online) Linear and nonlinear propagation of Airy pulses. (a) Evolution of the main hump in the linear case, (b) and (c) spectral components of the pulse depicted in panel (a) for anomalous (b) and normal (c) dispersion, (d) and (e) spectral distribution for anomalous (d) and normal (e) dispersion, (f) and (g) nonlinear temporal evolution corresponding to (d) and (e), respectively. Here, the dashed lines show the linear trajectories.
The dashed white lines in Figs. 2(b) and 2(c) are plotted based on the above formula and represent the spectral shift for the main hump (in a similar fashion to what is expected for Airy beams from the caustic theory [5]). If we only consider the intense hump, Airy pulse propagation can be modeled as that of a chirped Gaussian pulse with a linearly varying carrier frequency along the propagation, as can be seen from Figs. $\underline{2(b)}$ and 2(c), respectively.

In a Kerr medium (i.e., $\eta \neq 0$ ), the nonlinear evolution of the Airy pulse in function of the propagation can also be approximately treated as a Gaussian pulse with a different carrier frequency []․ Similar to the results in Figs. 1(a) and 1(b), the spectrum exhibits a spectral notch and peak when propagating in a regime of anomalous and normal dispersion, respectively. Since the frequency for the main Airy hump changes with the propagation distance, the defects induced by a mild nonlinearity also shift accordingly, as shown in Figs. 2(d) and 2 (e). In the spectral domain, their paths are expected to follow the analytical shift $v_{d}$ (dashed lines), but some deviations occur due to the nonlinear nature of pulse propagation and such deviations are more pronounced for the normal dispersion case, as clearly shown by studying the evolution in the temporal domain [see Figs. 2(f) and $2(\mathrm{~g})]$. The main hump tends to shrink (expand) for the anomalous (normal) dispersion case, indicating a steeper (slower) acceleration, which is analogous to the nonlinear dynamics of Airy beams under a self-focusing (defocusing) nonlinearity in the spatial case $[10,13,14]$.

From further numerical investigations at even higher, yet still mild, pump intensities (data not shown) we conclude that the spectral evolution of the peak/notch follows the prediction of Eq. (4), corrected by a parameter $\varepsilon$ that is slightly smaller than 1 in the case of normal dispersion, and is close to 1 for the anomalous dispersion, i.e., $v_{d}^{\prime}=\varepsilon v_{d}$. The direct outcome of this analysis is that the spectral defect shifts in frequency along the fiber and can be controlled by merely shifting the initial cubic phase mask in a fiber with a fixed length $L$ as follows: $v_{d}^{\prime}\left(v_{m}\right)=\varepsilon v_{m}+\varepsilon \operatorname{sgn}\left(\beta_{2}\right) L / 2$, where the shift of the spectral defect is proportional to the offset of the cubic phase.

To realize the spectral control described above, we use the experimental setup shown in Fig. 3. The input pulses come from a wavelength-tunable subpicosecond fiber laser from Pritel, Inc. Their spectra are then modulated by a cubic phase structure $\left(\omega_{s}=2 \pi \times 10^{11}\right)$ produced in a pulse shaper where the so called space-to-time pulse shaping technology is employed. The output Airy pulse is subsequently amplified by an erbium-doped amplifier and sent into a nonlinear fiber. An optical spectrum analyzer (OSA) is used to measure the output spectra.

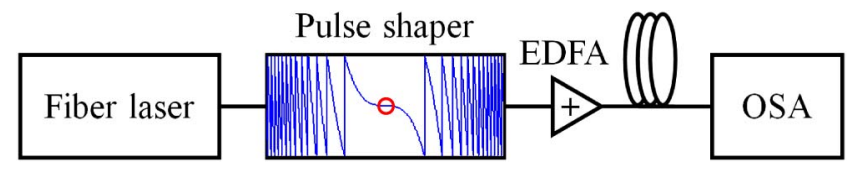

Fig. 3. (Color online) Experimental setup. EDFA, erbiumdoped fiber amplifier and OSA, optical spectrum analyzer. The plot in the pulse shaper schematically shows the cubic phase structure wrapped between $-\pi$ and $\pi$ where the circle indicates its center. 


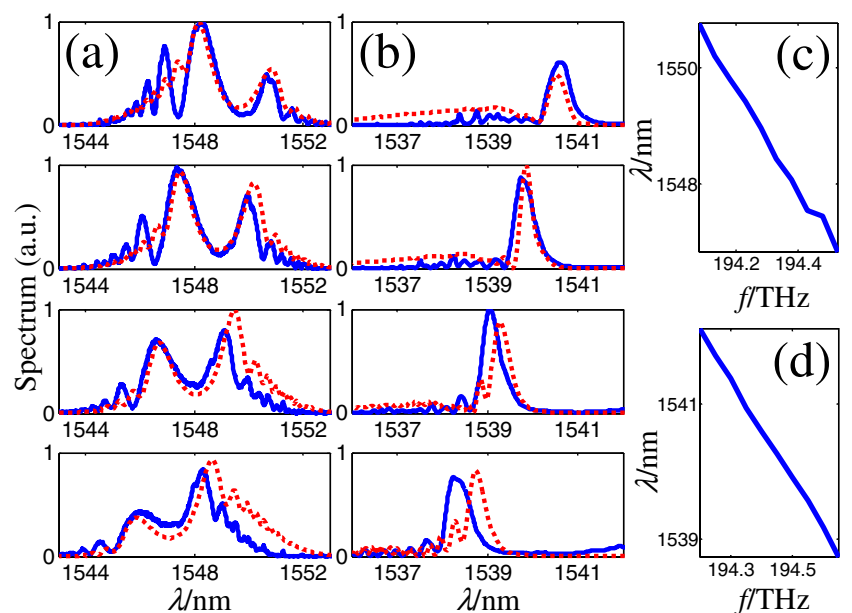

Fig. 4. (Color online) Experimental results (blue solid curve) and theoretical prediction (red dashed curve) of frequency shift control in (a) LEAFs and (b) DSFs. (c) and (d) plot the positions of the spectral notch and peak relative to the center of the cubic phase structure, corresponding to (a) and (b), respectively.

We performed the first experiment in a $5 \mathrm{~km}$ large effective area fiber (LEAF). In order to obtain anomalous dispersion, the fiber laser was tuned to generate subpicosecond pulses with a $1548.5 \mathrm{~nm}$ central wavelength and a bandwidth of $\sim 4 \mathrm{~nm}$. After the amplification, the recorded average power was nearly $240 \mu \mathrm{W}$. The corresponding spectra measured by the OSA are shown in Fig. 4(a). It is clear that the spectrum (solid lines) concentrates mainly into two major peaks separated by a notch. We observe that by shifting the center of the cubic phase structure to the frequency $f=194.2,194.3,194.4$, $194.5 \mathrm{THz}$, the notch gradually shifts from longer wavelengths to shorter ones. Figure $\underline{4(\mathrm{c})}$ plots the notch position with respect to the center of the cubic phase structure. From a linear fit, we obtain a value for the correction parameter $\varepsilon$ of 1.07 , which is close to what we expect. The dashed lines in Fig. 4(a) show the results obtained by simulating the nonlinear propagation with the input parameters corresponding to the experimental setting, which reproduce our experimental findings.

In a second set of experiments, we studied the nonlinear pulse propagation in the case of normal dispersion. We tuned the laser to produce pulses with a central wavelength of $1538.7 \mathrm{~nm}$ and a bandwidth of $4.7 \mathrm{~nm}$, which were then injected in a $6.6 \mathrm{~km}$ dispersion-shifted fiber (DSF) with a zero dispersion wavelength of around $1559 \mathrm{~nm}$. After amplification, the power at the input of the DSF is around $630 \mu \mathrm{W}$. We observed that following nonlinear propagation, the spectrum mainly concentrates into one peak surrounded by tiny ripples. Likewise, by shifting the center of the cubic phase structure to the frequencies $f=194.35,194.45,194.55,194.65 \mathrm{THz}$, the position of the single peak moves from longer wavelengths to shorter ones [Fig. 4(b)]. The relationship between the single peak position and the center of the phase structure is almost linear as shown in Fig. 4(d), where $\varepsilon$ is around 0.93 as inferred by the experimental data. The spectral distribution expected from the numerical model is depicted in
Fig. 4(b) (dashed curve). In this case, the shift rate $(\varepsilon \approx 0.73)$ is smaller than what we observed in the experiment, but it is still consistent, especially considering that no fitting parameters have been employed.

In summary, we have demonstrated the nonlinear spectral reshaping of Airy pulses in fibers into one or two self-shifting peaks for normal and anomalous dispersion, respectively. Different wavelengths can be selected by simply shifting the corresponding cubic phase structures. Since the propagation length is mapped into the frequency domain, metrological applications can be readily envisaged. Similarly, such a nonlinear spectral reshaping is also expected for nonparaxial diffraction-free beams [든 $\underline{17}]$.

This work is supported by the NSERC (Natural Sciences and Engineering Research Council of Canada) Discovery and Strategic grant programs, and by US National Science Foundation and AFOSR. Y. Hu acknowledges support from MELS FQRNT Fellowship. M. Clerici acknowledges the support of IOF People (Marie Curie Actions), EU FP7-2012, KOHERENT, GA 299522.

\section{References}

1. G. A. Siviloglou and D. N. Christodoulides, Opt. Lett. 32, 979 (2007).

2. A. Chong, W. Renninger, D. N. Christodoulides, and F. W. Wise, Nat. Photonics 4, 103 (2010).

3. D. Abdollahpour, S. Suntsov, D. G. Papazoglou, and S. Tzortzakis, Phys. Rev. Lett. 105, 253901 (2010).

4. I. Kaminer, Y. Lumer, M. Segev, and D. N. Christodoulides, Opt. Express 19, 23132 (2011).

5. Y. Kaganovsky and E. Heyman, J. Opt. Soc. Am. A 28, 1243 (2011).

6. Y. Fattal, A. Rudnick, and D. M. Marom, Opt. Express 19, 17298 (2011).

7. C. Ament, P. Polynkin, and J. V. Moloney, Phys. Rev. Lett. 107, 243901 (2011)

8. G. A. Siviloglou, J. Broky, A. Dogariu, and D. N. Christodoulides, Phys. Rev. Lett. 99, 213901 (2007).

9. Y. Hu, G. Siviloglou, P. Zhang, N. Efremidis, D. Christodoulides, and Z. Chen, in Nonlinear Photonics and Novel Optical Phenomena, Z. Chen and R. Morandotti, eds. (Springer, 2012), pp. 1-46.

10. Y. Hu, Z. Sun, D. Bongiovanni, D. Song, C. Lou, J. Xu, Z. Chen, and R. Morandotti, Opt. Lett. 37, 3201 (2012).

11. G. P. Agrawal, Nonlinear Fiber Optics, 3rd ed. (Academic, 2001).

12. Y. Hu, P. Zhang, C. Lou, S. Huang, J. Xu, and Z. Chen, Opt. Lett. 35, 2260 (2010).

13. I. Kaminer, M. Segev, and D. N. Christodoulides, Phys. Rev. Lett. 106, 213903 (2011).

14. A. Lotti, D. Faccio, A. Couairon, D. G. Papazoglou, P. Panagiotopoulos, D. Abdollahpour, and S. Tzortzakis, Phys. Rev. A 84, 021807(R) (2011).

15. I. Kaminer, R. Bekenstein, J. Nemirovsky, and M. Segev, Phys. Rev. Lett. 108, 163901 (2012).

16. F. Courvoisier, A. Mathis, L. Froehly, R. Giust, L. Furfaro, P. A. Lacourt, M. Jacquot, and J. M. Dudley, Opt. Lett. 37, 1736 (2012).

17. P. Zhang, Y. Hu, D. Cannan, A. Salandrino, T. Li, R. Morandotti, X. Zhang, and Z. Chen, Opt. Lett. 37, 2820 (2012). 